\title{
Proprioceptive Shape Signatures for Object Manipulation and Recognition Purposes in a Robotic Hand
}

\author{
Alex Vasquez ${ }^{\mathrm{a}, *}$, Veronique Perdereau ${ }^{\mathrm{a}, * *}$ \\ ${ }^{a}$ Sorbonne Universités, UPMC Univ Paris 06, CNRS, ISIR, 4 Place Jussieu, 75252 Paris, France
}

\begin{abstract}
Tactile information has been largely exploited for object recognition with robotic hands but very few approaches have used proprioception alone. In those that do, raw values of joint angles or torques are exploited to train learning algorithms. However, these approaches under-exploit the potential of proprioception, such as its usefulness to estimate the object pose and size. Furthermore, they focus on recognizing individual objects, which increases the amount of data needed to train the algorithms. In this paper, we present an approach based only on joint angles of a robotic hand to generate a shape proprioceptive signature that is invariant to the size and position of the object. Instead of recognizing a specific object from a list, object characteristics useful for its manipulation are extracted. This signature is exploited not only for shape recognition but also for pose estimation. To illustrate the scope of this method, tests are performed on primitive shapes. Results show that the signatures are invariant within large ranges of sizes and poses. Experiments on real hand were carried, and results depicted that the method works similarly in both simulated environment and real applications. A comparison between this two results is made and discussed.
\end{abstract}

Keywords:

Tactile Sensing, Multi-fingered Robotic Hand, Object Recognition and Manipulation

\section{INTRODUCTION}

When identifying the shape of an object, human beings make a combination of visual and tactile data mainly. All the information coming from the related senses allows us to identify lots of characteristics of the objects we manipulate with a remarkable accuracy of about the $94 \%$ and a rapidity of less than 5 seconds [1]. In an intent to reproduce such a performance with robotic manipulation systems, vision is the sense that has received most attention and has been largely exploited for object shape identification. In this sense, vision-based approaches for object shape identification perform a geometric model reconstruction (GMR). Wang et al [2] used a laser to scan the object and obtain a cloud point for 3D shape reconstruction. Jang et al. [3] used a stereo camera to get both an image that was analyzed to recognize the object and a cloud point that will be replaced with a prerecorded 3D model of the object once the object has been recognized. Furthermore, Lippierllo et al. [4] introduced a could point which adapted to the shape of the object as this was explored with a camera from different angles. Regardless the good results presented in cited works, they still present some limitations: First, online application is questionable due to the computational burn and time to complete a proper estimation of the 3D model, second, occlusion can easy occur once the object is grasped. Tactile data, on the other hand, is not subjected

\footnotetext{
*Corresponding Author

Email addresses: vasquez@isir.upmc.fr (Alex Vasquez), veronique.perdereau@upmc.fr (Veronique Perdereau)
}

to occlusions and can provide data about both the shape and location of the object when the object is grasped.

Works on tactile object identification have increased over the years and the evolution of sensor technology has allowed to make good progress in that area, but there is still a lot of research to be done. In robotics, many types of sensors have been embedded on hands of manipulators to reproduce the sense of touch $[5,6,7,8]$ and most of literature in the domain mainly focuses on identifying the geometrical properties of objects using these sensors $[9,10,11,12]$. However, according to Lederman et al. [13] when tactile object identification is to be performed, both tactile and proprioceptive information are involved. Whereas there are many methods using tactile information, proprioception is far from being exploited. Attempts to take its advantages on robots for tactile object recognition are seldom found in the literature. When found, proprioception is often combined with other sensing modes such as touch $[14,15,16,17]$, vision [18] and hearing [19]. Although proprioception is largely used by humans to perform shape identification, it has been little exploited as the only source of information by robotic systems. Furthermore, a great number of robotic hands do not have enough embedded tactile sensors to perform object recognition but they do have sensors to measure and control the joint angles of its fingers [20, 21, 22]. Thus, proprioception is their more reliable source of information for haptic shape identification.

Few studies have used proprioceptive information alone to perform object recognition and most of them use raw values as inputs to their identification algorithm. For instance, Faria 
et al. [23] correlated hand configurations during in-hand object exploration to generate hypotheses on a potential candidate from a set of objects. Johnsson [24] and Ratnasingam [25] implemented Self-Organizing Maps (SOM) based on joint angle measurements of a robotic hand recorded when grasping an object. Bergquist et al. [26] also implemented SOM, but instead of joint angles, used joint torques recorded during interactions with the objects like lifting, shaking, dropping, crushing and pushing. Okamura et al. [11] used both tactile and proprioceptive data to identify a group of shape patterns during tactile exploration and concluded that tactile information is not needed to recreate object shape.

Most of these methods achieve good results with accuracy rates higher than $85 \%$. However, inasmuch as they use raw values of the joint angles/torques, they all require training sets to be large for the recognition algorithms to attain such results. Furthermore, even if these approaches are tested on a large number of objects, most of these objects have the same global shape. This demands several samples of each individual object in the training set which enlarges its corpus, thereby the time to record its data. This also has a detrimental effect on the performance of the recognition algorithm when new objects are added to the set. Finally, most of the existing methods do not provide with a methodology to estimate any information about the pose of the object within the hand.

In this paper, we present a new approach to extract information about the global shape of the object solely based on proprioceptive data coming from the robotic hand. The main contributions made in this work can be listed as follows:

- Instead of using the raw values of the joint angles, we propose to represent them as proprioceptive signatures. These signatures correspond to the descriptor that we propose in this article to represent the shape of the object using the hand configuration.

- Thus, shape information contained in the joint angles become invariant to the pose and the size of the object within the hand. These signatures are generated from a representation of the joint angles of the hand based its kinematic topology to endow the hand with proprioception. This mimics, to some extent, the human proprioception which combines joint angle information with a representation/kinematic model of their body in their mind [27].

- We focus on the global shape identification instead of specific object recognition, in contrast to the cited methods exploiting proprioception. To do so, we exploit the proprioceptive signatures generated in this method. This is advantageous since, in non-structured environments, many objects might share the same geometrical properties which makes it more difficult for the learning algorithm to discriminate them.

- The amount of data needed to train the learning algorithm is reduced with respect to the proposed methods using proprioceptive information. This is a good asset because data gathering for tactile object recognition could be tedious. Furthermore, a possibility of using synthetic data to this purpose is presented, which is beneficial when the simula- tion model of the robotic hand is available.

- Shape can be identified after performing a single grasp. This offers an advantage over the tactile-based or visionbased methods which, most of the times, need several samples before being able to recognized an object. Runtime measurements will be presented in the experimental part of this article.

- Estimation of the pose of the object is possible using the signatures, which has not being done by proprioceptionbased methods.

This paper begins with defining the proprioceptive representation of a robotic hand used in this approach. Next, the conditions are defined for this representation to contain information about the shape of the object. This information is described as a signature that depends on the geometry of the object only. Then, the analysis of the finger configurations is presented as a tool for shape recognition. Finally, simulated results are presented using a Shadow Hand and the iCub Hand along with experimental results obtained with a Shadow Hand.

\section{METHOD}

This section is dedicated to explaining the methodology of the presented approach. First, the procedure followed to create the representation of the joint angles of a robotic hand is explained to provide the hand with proprioception and make it able to perform object shape identification. Then, it is shown how the shapes generate patterns, called signatures, from this representation. This is exemplified on a set of primitive shapes. Finally, it is illustrated how the information about the fingers configurations during the grasp closure is also exploited to obtain more information about the shape of the object. Features from the set of test objects are finally extracted to identify the different test shapes.

\subsection{Proprioception for Object Shape Information}

When an object is grasped, both tactile and proprioceptive modes can provide information about its shape. The relevance of the information provided by each of the modes is variable and depends on the geometrical properties of the object and the hand as well as the exploration procedure followed. One of the properties of the object that most in uences how relevant these modes are is its size. When the size of the object requires the whole hand to be manipulated, proprioception becomes an important input for shape identification [28]. This finding is the rationale behind the design of this new method because it intends to exploit proprioception as a useful source of information on the shape of the object.

The other factor contributing to how the shape information provided by proprioception is useful is the exploration procedure. According to Lederman et al. [13], the enclosure grasping is the exploration procedure employed by most individuals for global shape identification. In this kind of grasp, the hand takes the object within its fingers adapting each finger to its shape. Thus, the configuration of the fingers and their relative positions contain information on the shape of the object. In this 


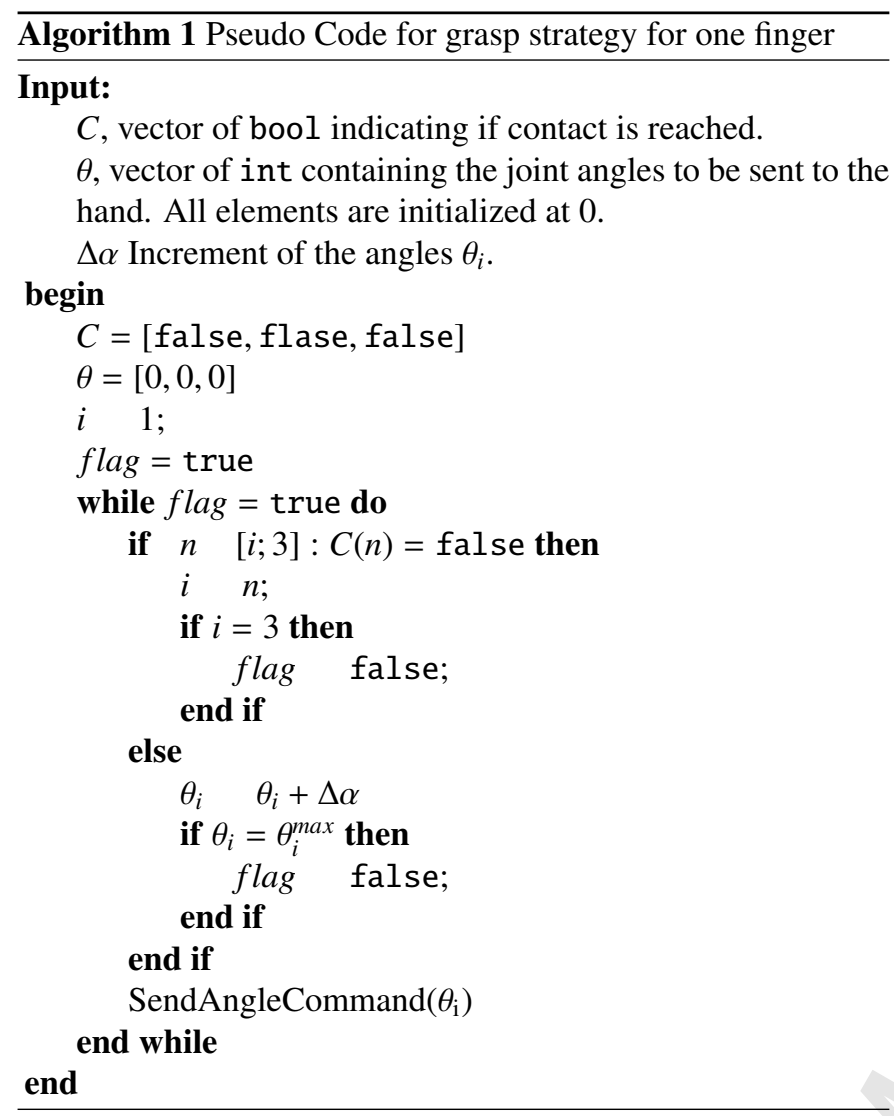

scenario, proprioception carries significant information about the shape of the object in the joint angles as their configuration describe, to some extend, the shape of the grasped object.

\subsubsection{Grasping Strategy}

To reproduce the enclosure grasping with a robotic hand, the proposed method uses the grasping strategy described in [15]. In this grasp, $\theta_{i}$ starts increasing until a contact $C_{i}$ is detected on any phalanx $n$ upstream in the chain ((Fig. 1 shows these parameters and what upstream refers to). If no contact is detected, the angle $\theta_{i}$ will continue to increase until reaching its maximum value $\theta_{i}^{\max }$ (this angle depends on the robotic hand used). Once $\theta_{i}$ stops, the same procedure is followed with $\theta_{i+1}$ and so on to $\theta_{I}$, where $I$ is the number of phalanges of the used hand. Naturally, $I=3$ for anthropomorphic robotic hands. Algorithm 1 shows this procedure otherwise. For this grasping strategy, it is necessary that at least the proximal phalanx is not coupled with the rest of the phalanges. Otherwise, a different grasp strategy might be needed.

\subsubsection{Shape Proprioceptive Signature}

In human beings, proprioceptive information comes from several sensory receptors located in the muscles, tendons and joints. In a robotic hand, the joint angles can be used to reproduce the information coming from these sensory receptors and provide the hand with proprioception.

When each finger conforms to the shape, the proprioceptive data coming from the hand is then exploited to extract information on the shape. Features like corners and edges are not identifiable without tactile information. As a result, any polygonal shape which cross-section provokes the joint angles to behave similarly generating the same information, and therefore, prorpioception alone cannot discriminate them. Thus, for the sake of simplicity without losing the generality, this method assumes that when the enclosure grasp is performed around an object, each finger adapts to a circular shape. As illustrated in Fig. 2, the central angle subtended by the arc formed by the finger on the circle is then equal to the angle between the phalanges forming that arc (See Appendix).

The underlying relationship between the joint angle $\theta$ and the arc length $s$ sets a link between the hand configuration and a geometric parameter of the object, namely the radius $r$ of the enclosed circle, as follows:

$$
s=r \theta
$$

Since the length of the finger is constant, it can be stated that the arc length $s$ is also constant. Thus, the angle $\theta$ and the radius $r$ become inversely proportional variables.

Consider two fingers adapting to two different circles but forming same arcs on each. Fig. 3a shows that the angle $(\varangle A O B)$ subtended by the arc on the larger circle is smaller than the one $(\varangle C O D)$ on the smaller circle. Given that the joint angle can be known, the radius $r$ of a circle can be inferred by (3). When this is applied to each finger, the radius of the different parts of the object shape can be obtained.

Each finger in contact to the object will generate a chain of arcs on the object surface. The set of chains created by every finger forms a discrete representation of the contact surface and the contact surface can be reconstructed through interpolation between the different chains. To obtain a smooth surface, a cubic interpolation is adopted, which confirms that small details on discontinuities on the surface of the object will not be represented there. Fig. 3b) illustrates the result of the interpolation between four circles of different radius. The blue manifold represents the contact region between the object and the fingers. This manifold is then exploited to generate the proprioceptive signature of the shape.

\subsection{Proprioceptive Data Representation}

To generate the proprioceptive signature of an object in the hand, a representation of the proprioceptive data of the robotic hand is first created. Based on the fact that human beings use

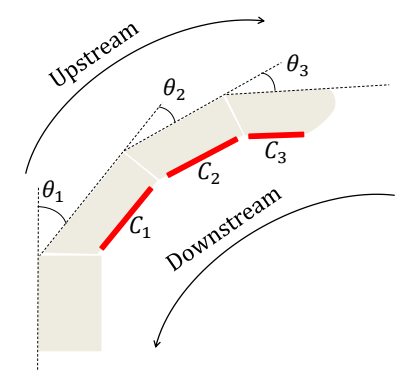

Figure 1: Lateral view of a finger. Read lines show the areas of contacts $C_{i}$ onto which the contacts are detected. $\theta_{i}$ are the joint angles. 
mental images of the joint angles to detect features of the objects [29], this method uses an image to represent the joint angles of the hand, therefore, the finger configurations. In this sense, we call it Finger Configuration Image (FCI).

Because FCI represents the proprioception of the hand, it is important that it respects the definition of proprioception itself which implies the knowledge of the position of the neighboring parts of the body relative to each other. In the case of the robotic hand, it should provide, to some extent, the relative positions of the segments (phalanges) of the fingers with respect to each other. To fulfill this requirement, FCI is created so that each one of its pixels corresponds to a joint, as illustrated in Fig.4.

The thumb is discarded from this representation because it is generally in an opposed position with respect to the rest of the fingers when an object is grasped [30]. Thus, the information integrated across the other fingers yields a better representation of the surface of the object than the information gathered from the thumb [31]. This makes the proprioceptive information coming from the thumb unreliable for shape identification.

\subsubsection{Finger Configuration Image}

The FCI is then filled with information based on the kinematic topology of the fingers, which refers to the description of the configuration of the links and joints. For this approach, each finger is considered as a serial manipulator. Hence, each joint angle is the sum of all joint angles downstream in the chain, the proximal phalanx being the first link in that chain. Based on this, the following equation is derived to fill the FCI using the joint angles $\theta$.

$$
F C I(y, f)=k \sum_{i=1}^{y} \theta_{i, f}
$$

where $F C I(y, f)$ pixel on the row $y$ and column $f$ of the image $F C I$ and it corresponds to the representation of the joint angle shown in Fig. 4. $\theta_{i, f}$ is the angle of the joint $i$ (row) on the finger $f$ (column). Fig. 1 illustrates these angles on one finger. $k$ is a transformation constant from angle to gray scale value. The value of $k$ depends on the bit depth of the pixels of the FCI. We define, generally this value using the following expression:

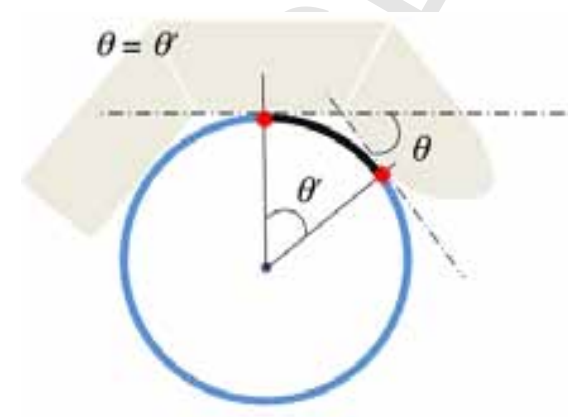

Figure 2: Lateral view of a finger conformed to a circle. The red points represent the contact points of the phalanges. These points form an arc shown in black that subtends the angle $\theta$. $\theta$ is the angle between the phalanges forming the arc on the circle.

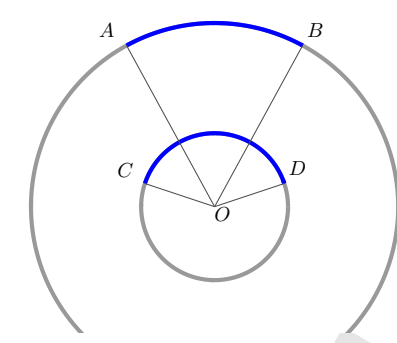

(a)

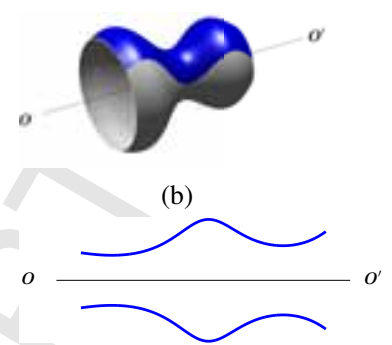

(c)
Figure 3: (a) Concentric circles showing the central angles of their corresponding intercepted $\operatorname{arcs}(\widehat{A B}$ and $\widehat{C D})$. Both arcs have the same length. (b) Continuous case of Fig. 3a. Infinite sequence of circles of different radius forming a shape (gray) and the manifold containing the arcs of the same length formed on each circle (blue). (c) Central angles subtended by their arcs contained in the manifold along the central segment $\overline{O O}$.

$$
k=\frac{C}{3}
$$

where $C$ is the maximum output level defined by the color precision in which the $F C I$ was defined (e.i., 256 levels for a 8-bit image). It is divided by three so that there is an equal distribution of the color among the three phalanges (under the assumption that the three joints share the same maximum value). The result is an image which pixels values are updated when the joint angles of the concerned fingers change. Fig. 5 presents two examples of how finger configuration is represented in the image $F C I$. The image on the left shows that when the phalanges of a finger are aligned, the pixels corresponding to that finger share the same gray scale value. On the other hand, the image on the right shows that aligned fingers are represented as rows with the same gray scale value.

\subsubsection{Proprioceptive Signatures from the FCI}

The FCI is used to generate the proprioceptive signature of the object once the grasp is achieved and the fingers conform to the shape. For this, a two-step procedure is followed. First, the FCI is increased in size using cubic interpolation which soften the transition between the different radius to which each finger adapts. Second, the signature $S(x)$ is then built based in this interpolated FCI ( FCI ) using the following expression.

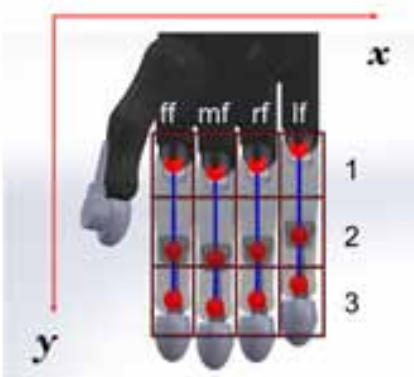

Figure 4: Image superposed to the hand so each pixel corresponds to one joint. Consequently, each column represents one finger and its pixels represent the joints of the finger. The thumb is not included in this representation. 


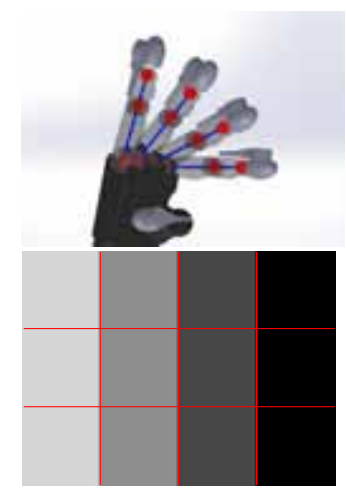

(a)

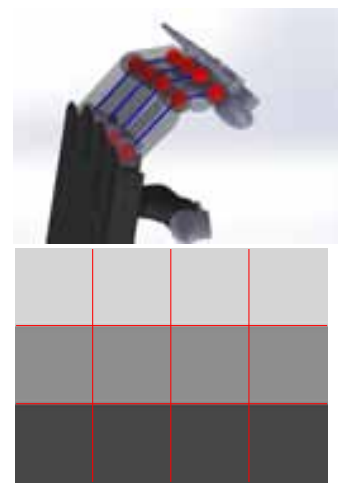

(b)
Figure 5: Finger configurations (Upper images) and their corresponding Finger Configuration Images (lower images). (a) Example of aligned phalanges and misaligned fingers. (b) Example of misaligned phalanges and aligned fingers.

$$
S(x)=\left\{\begin{array}{l}
-\min \mathrm{FCI}_{x} \\
\max \mathrm{FCI}_{x}
\end{array}\right.
$$

which allows plotting two lines based on the minimum and maximum gray scale values in the corresponding column $x$ of the FCI . Because most robotic hands have coupled joints on the fingers, this representation integrates the information of those joints intrinsically. Thus, the lower line of the signature represents the angle of the proximal phalanx and the upper line represents the addition of the rest of angles.

\subsection{Temporal Finger Configuration Analysis}

As the hand is molded onto the shape of the object when it closes around it, the finger closing movements for each shape re ects differently on the FCI. The grasp strategy also enables to predict the fingers that might not be in contact with the object. This section shows how the FCI is exploited to this end.

\subsubsection{FCI temporal analysis}

The grasp strategy used in this approach establishes a sequential change of the joint angles for each finger starting from the closest to the palm. This sequence is re ected in the FCI. As expressed in (2), the gray scale values of the pixels of the FCI depend on the angles of the joints downstream in the chain. Thus, all pixels of each column will share the same gray scale value as long as the finger has not entered in contact with the object. Once the contact is made by a phalanx, the upstream joint will start changing its angle sequentially which makes the pixels of the same column have different gray scale values.

These variations in the grasp closure at finger level can be detected in the FCI by computing the difference between the maximum and minimum gray scale values of each column. These differences are periodically computed during the grasp execution. The vectors of gray scale values resulting from these computations are stored in the rows of a matrix $G$ as follows:

$$
G\left(y_{t}, x\right)=\max _{x}\left(F C I_{t}\right)-\min _{x}\left(F C I_{t}\right)
$$

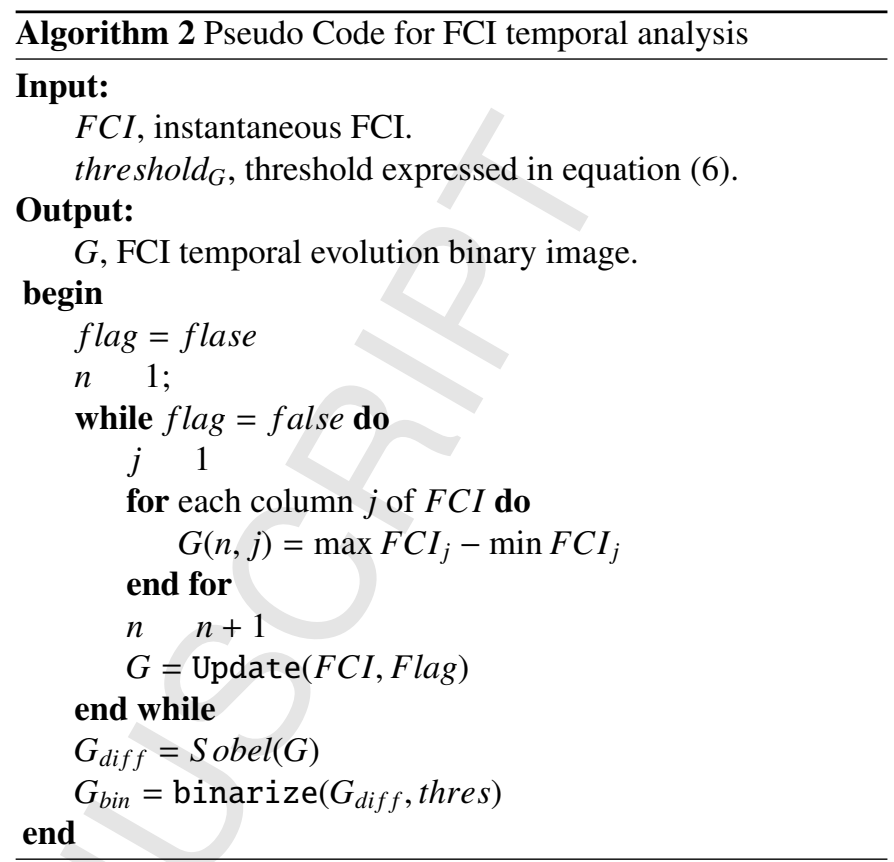

where the right term is the difference between the maximum and minimum gray-scale values in each column $x$ of the FCI at the instant $t$ during the grasp execution. Each time this difference is computed for each column $x$ over time, the obtained vector is stored in the row $y_{t}$ of $G$. In $y_{t}$, the subscript $t$ indicates its value increases as the time passes.

When one of the fingers has already finished adapting to the object, the computation of (5) is stopped. Because the increase of the angles of each finger are re ected on the image $G$ as changes of the gray scale value on the corresponding rows, a Sobel filter is applied to this image in order to compute derivatives on the vertical direction. Then, the image is binarized with a threshold Threshold $_{G}$ computed using (6) (Algorithm 2).

$$
\text { Threshold }_{G}=0.75 \times 8 \times \Delta I_{\theta}
$$

where the constant 8 comes from the Sobel operator and $\Delta I_{\theta}$ corresponds to the changes of the gray scale values of the FCI or joint angles $\theta$ at the frequency the image $G$ is sampled. The threshold is defined to be $75 \%$ (which explains the constant 0.75 ), to compensate for the inexactitudes of sampling synchronization.

\subsubsection{Non-Contact Fingers Drop-out}

To this point, it has been assumed that all fingers were in contact with the object. This poses a problem when the object is too small for all fingers to be in contact with it. In general, it poses a problem whenever one of the fingers does not make contact with the object.

If the prior situation occurs, the signatures would not correspond to any of the patterns expected for a given shape. Hence, the information about the fingers that are not in contact should be ignored before generating the signature.

This method makes use of the FCI temporal analysis to detect the fingers that are not in contact with the object. For those fin- 

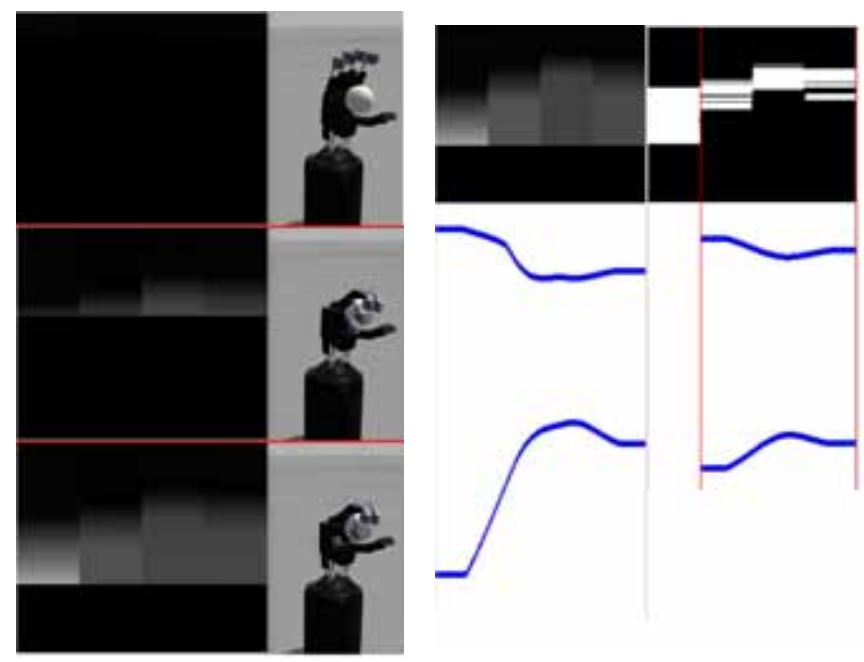

(a)

(b)

Figure 6: (a)Generation of the image $G$ overtime. As the hand adapts to the object, the rows of $G$ are been filled with the difference between the minimum and maximum gray scale value of the columns of the FCI. (b) Once it has been detected that the little finger did not adapt to the object, the generated signature (left) is corrected by droping out the information coming from that finger (right).

gers, the joint angles do not change until the downstream ones reach their maximum values. This is re ected in the binarized image $G$ by the white areas which are used to detect the fingers that do not adapt to the object shape. In this sense, the position and the length of this white areas are computed for each column. To determine if a finger did not adapt to the shape, a threshold of these two parameters are set.

To estimate the values of these thresholds, the grasp strategy is executed with no object within the hand. Once algorithm 2 has been executed, the length and position of the white areas are computed for each finger and the threshold for each parameter is chosen to be $85 \%$ of the computed value. In case the middle or ring finger are detected as not-in-contact fingers, it is assumed that an error has occurred in the process of grasp execution. Algorithm 3 shows the pseudo code to dropout the information of the finger. Furthermore, Fig. 6 shows how images of this procedure along with a corrected signature.

The process of dropping out the information from noncontact fingers contributes to generating proprioceptive signatures that are not affected by the fact that one finger might not be in contact with it. However, to achieve this goal, further treatment is needed. The next section presents how signatures are processed so that they become invariant to size and pose of the object.

\subsection{Signature Enhancement}

Signatures can be subjected to changes depending on the size and pose of the object with respect to the hand. To make the signatures invariant to these parameters, the signatures are further modified so that the effect of each parameter is canceled.

First, the length of the signatures varies according to the temporal configuration analysis during the grasp execution. When some information is dropped out from the FCI, the length of $\overline{\text { Algorithm } 3 \text { Pseudo Code to drop out information from non }}$ contact fingers before signature generation

\section{Input:}

$G$, FCI temporal evolution binary image.

thres pos , Threshold for position of the white area.

thres length, Threshold for length of the white area.

\section{Output:}

$S$, proprioceptive signature.

\section{begin}

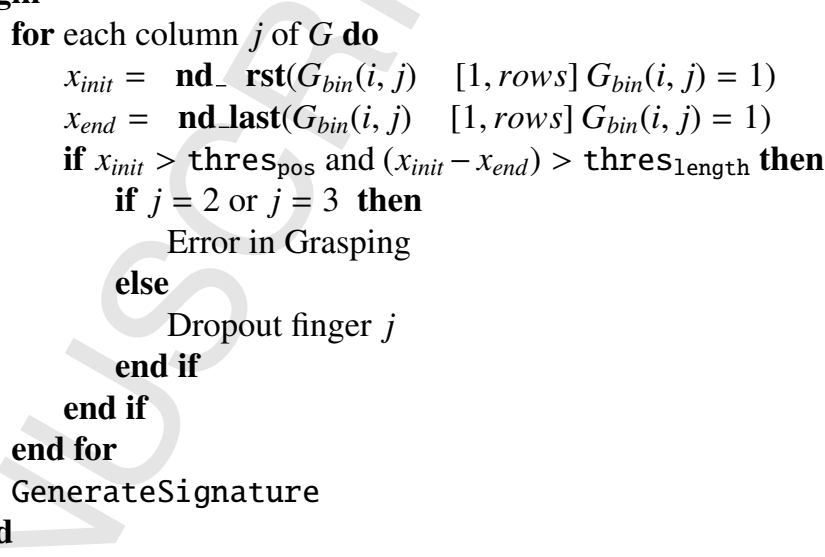

the generated signature is reduced (Fig. 7a). To later compare the obtained signatures with generic shape signatures, all signatures must have the same length. Thus, they are re-interpolated to a fixed length $m$ (Fig. 7b).

Second, the effect of the object size on the signatures is corrected. Because this parameter causes the signature to be shifted vertically, a further processing phase will center it with respect to the horizontal axis. To this end, each line of the signature is treated separately. The mean of all points corresponding to each line is subtracted to each point of the line (Fig. 7c).

Rotation of the object also affects the signature by inclination of it. This might lead to some resemblance between signatures of different objects. To correct this, the re-interpolated and centered signature is rotated (Fig. 7d) and its integral is computed for each angle $\alpha$. When a local minimum is found, the process stops and the signature is rotated this angle (Fig. 7e).

Finally, the signature is normalized between the range [-1 1$]$ (Fig. 7f). After this 3-step process, the effects of both the size and pose of the object have been discarded.

\section{EXPERIMENTS}

\subsection{Simulation}

This method was tested using the Gazebo simulator. The Gazebo model of the Shadow Hand was implemented. To outline its performance, the proposed approach was tested on a set of five primitive shapes (cone, torus, sphere, one-sheeted hyperboloid and cylinder). These shapes were chosen considering their frequent presence in household objects and some of them because they were not considered in previous related works. Some of these shapes were also chosen based on their geometrical properties, such as convexity. Fig. 8 shows these shapes 


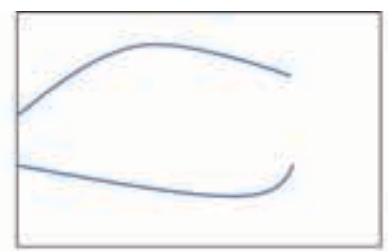

(a)

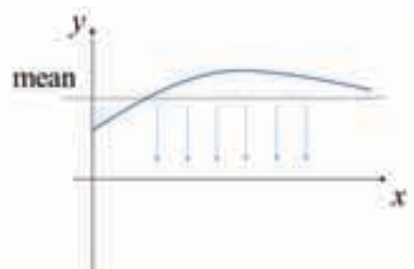

(c)

(e)

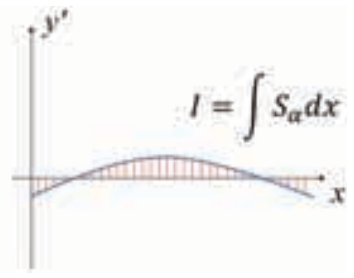

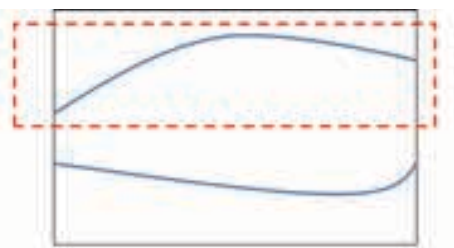

(b)

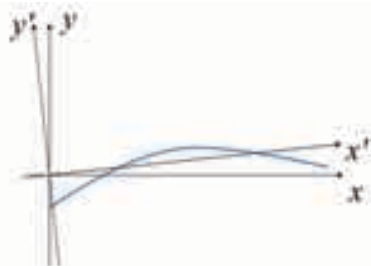

(d)

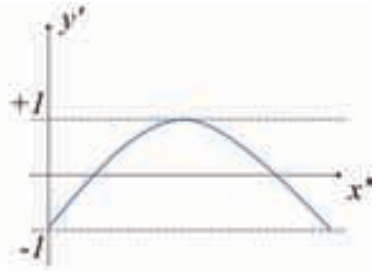

(f)
Figure 7: (a) Signature corrected by dropping out the information coming from the first finger. Therefore, its horizontal length is shorter. (b) Signature interpolated so that its length is equal to a non-corrected signature $m$. For the following steps, lines are treated separately. The upper line is used as example in this figure. (c) In order to correct the vertical shift due to the size of the object, the mean value of the signature is computed and then subtracted from every point to center the signature on the $x$-axis. (d) In order to correct the rotation, the signature $S$ is rotated on the coordinate system. (e) For each angle $\alpha$ of rotation, the integral $I$ of rotated signature $S_{\alpha}$ is computed (striped area). This process is repeated until the minimum integral is found. (f) Once the rotation is corrected, the signature is normalized between $[-1 ; 1]$.

with their corresponding generated signatures below. These shapes were simulated as static which means that the physics of the simulated world do not apply to them and their position is thereby not changed by the interaction forces with the hand.

\subsection{Data Collection}

To evaluate this method, the signatures of the shapes of different sizes and located in different parts along the work space of the hand were recorded. These sizes and locations are shown in table I. The range of each parameter was chosen to be extreme, so that proper boundaries were defined according to the resemblance between signatures. These ranges are given in Table I. A simulation included 10241 signatures combining all sizes and locations for each shape and to the ten rotation angles tested on the torus, hyperboloid and cylinder (The rotation angle tested can be visualized in Fig 10).

\subsection{Real-hand experiments}

The Shadow Hand was used to test this method in a real application. Real objects with the same primitive shapes of the simulated ones in section 3.1 were used (Fig. 9).

In the data collection process, between 4 and 7 signatures were collected for each object. Objects were handled to the

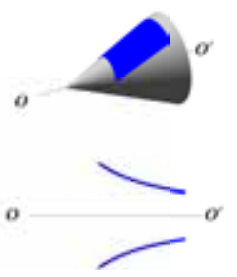

(a)

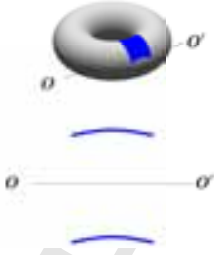

(b)

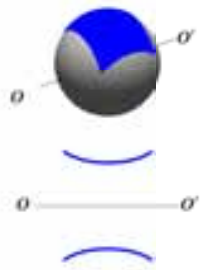

(c)

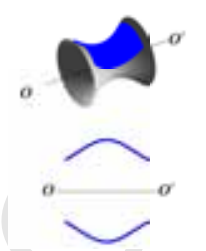

(d)

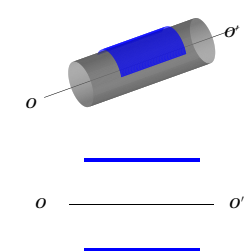

(e)
Figure 8: Set of test shapes. The blue manifold on top of them is the result of equivalent arcs formed on the circles contained in the shape. Below each shape, the corresponding signature obtained by extracting the central angles from the manifold with respect to the central axis $\overline{O O}$. The procedure shown in Fig. 3.

hand and they were free to move with the interaction forces with the hand during the grasp execution. A picture was taken after the grasp was executed, and this image was used to estimate the rotation angle of the object. This was done by computing the angle between axis of the hand and the object (See Fig. 10).

\subsection{Signatures Comparison}

Once the signatures were recorded, they were corrected as explained in Section 2.4 and compared to the theoretical ones. This was done using the Dynamic Time Warping (DTW) algorithm [32] which allows to perform shape analysis on the signatures and determine how similar the measured signatures were to the theoretical ones.

As a measure of similarity, DTW gives a distance-like measure between two sequences. This measure can be used to distinguish a signature from another. For this, a distance threshold was defined by comparing all the collected signatures with the expected patterns of the shape. The threshold value corresponds to the situation when all signatures match with their shape pattern. This threshold was later used to define the proper ranges.

\subsection{Defining Ranges for Size and Position}

The ranges within which the presented method works best were defined based on the results obtained from comparing the signatures to the expected ones. Given a shape, the probability of generating the correct signature was computed for each tested size and pose. These probabilities were computed from a set of simulations.

These probabilities were put into a matrix which dimensions depended on the number of variables changing during the tests. For instance, in the case of the Cone, only the radius and the vertical position changed, the matrix is therefore two dimensional. Once this was done, the mean of the probabilities of every continuous interval for each dimension of the matrix were computed and only those in which the mean was higher than $80 \%$ were kept. Then, the largest intervals from the chosen 


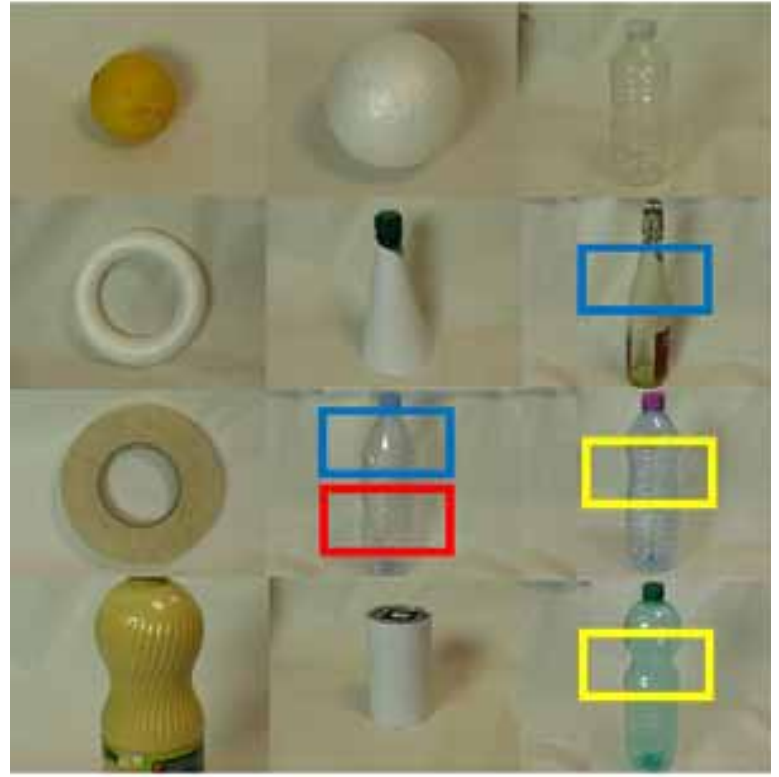

Figure 9: Objects used for real experiences. Some of the objects were grasped on different sites to get a different shape.This is indicated by rectangles on those objects. Yellow is used for hyperboloid, red for cylinder and blue for cone.

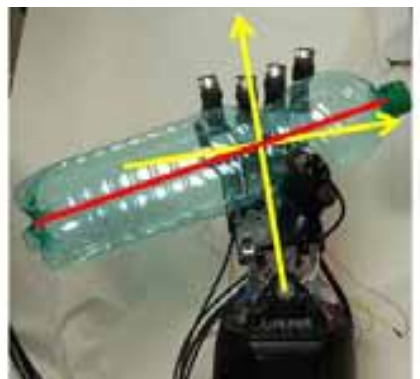

Figure 10: Object angle estimation. Yellow lines correspond to the hand axis. Red line to the object axis. The angle of rotation is measured between the horizontal axis of the hand and the axis of the object.

ones were considered to re ect the best possible performance of this method.

\section{RESULTS}

\subsection{Signatures from the FCI}

Fig. 11 shows the proprioceptive signatures obtained in simulation and with real objects prior to enhancement. It can be observed that they do not exhibit significant changes when the size or position of the shapes changed. As expected, a vertical and horizontal shifts are shown depending on the size and position, respectively. The bigger the object, the larger the distance between the lines conforming the signatures. Rotated objects are not shown in this figure.

Regardless their resemblance to the theoretical signatures, some differences are also observable. For instance, the signatures obtained for the cylinder have a certain similarity with the ones obtained for the hyperboloid (Fig. 12e). This is due to the kinematics of the hand and how the fingers are positioned with
Table 1: Tested dimensions and positions for each object. The column Shape Dimension shows the dimensions varying for each shape. In the column Size, the intervals of dimensions that were tested and the change rates. Images in the column Position correspond to the positions where the shapes were located. All dimensions are shown in $\mathrm{cm}$.

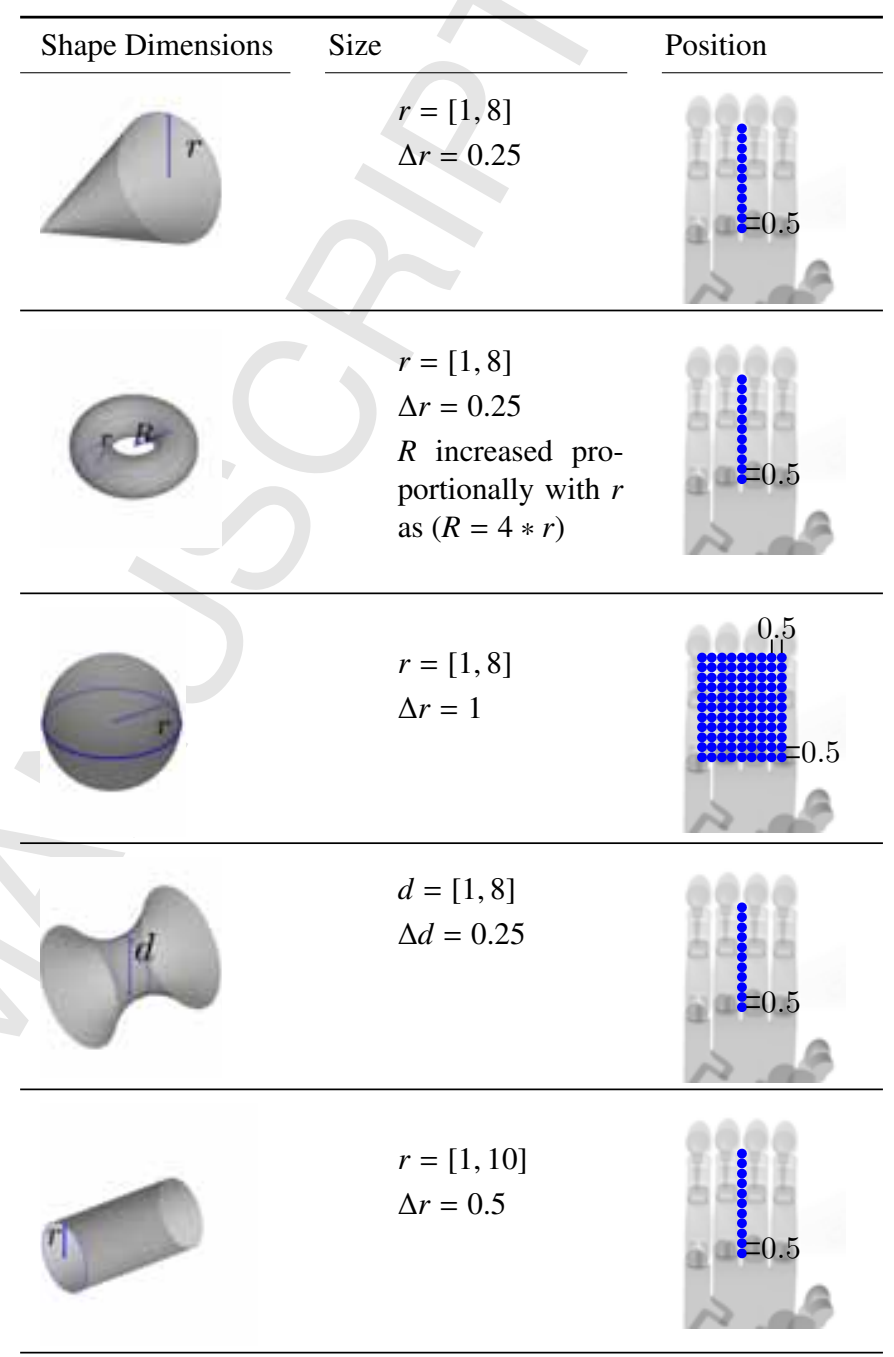

respect to each other, which was not considered in the FCI. Fig. 12 illustrates the signatures obtained for each shape after the signature enhancement procedure has been performed on the measured signatures. Signatures of the same shape show significant similarities regardless the changes of sizes of the object and the locations were put in the hand. This depicts, to some extend, their little variance with respect to these two parameters.

\subsection{Accumulated Distance Threshold}

The results of the procedure defining the threshold for the DTW algorithm (Section 3.4) are shown in Fig. 13a. These results were obtained in simulation. As can be observed, most of the shapes showed lower mean distance when compared to their corresponding theoretical signature but the cylinder did not show the same behavior. The reason for this is that the normalization made in the signature enhancement procedure makes its signature similar to the one of an hyperboloid. The 


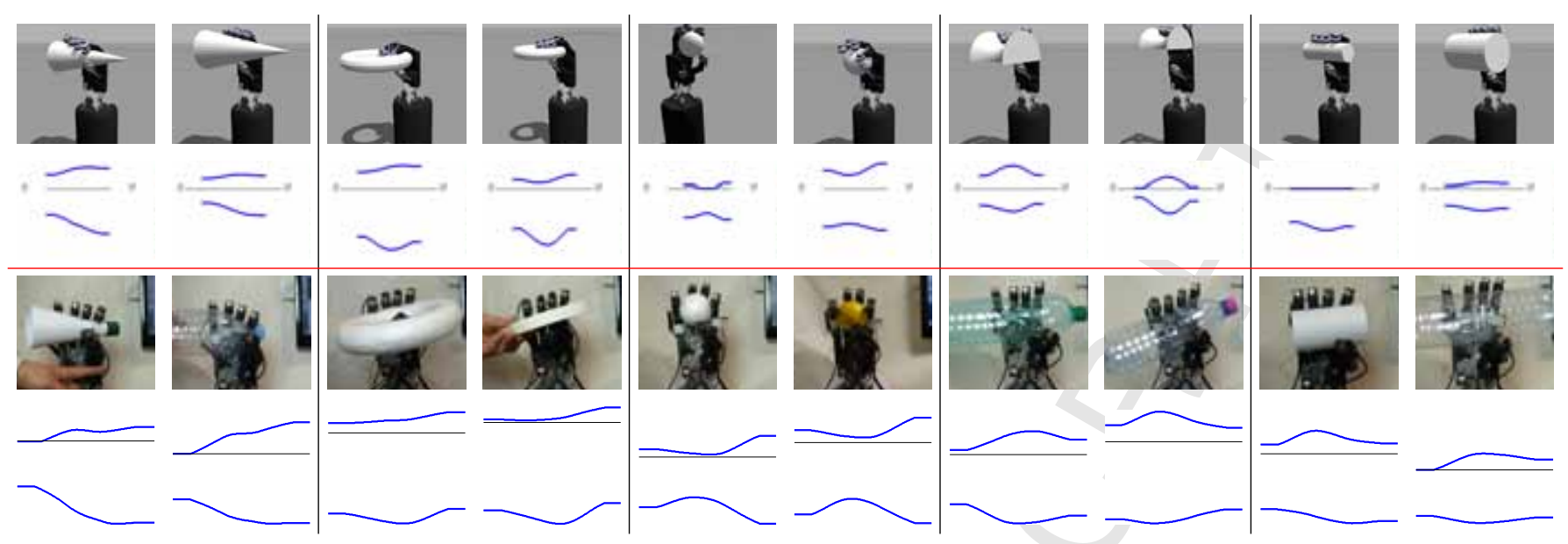

Figure 11: Signatures obtained on both simulated (upper) and real objects (lower). from left to right: cone, torus, sphere, hyperboloid, cylinder.

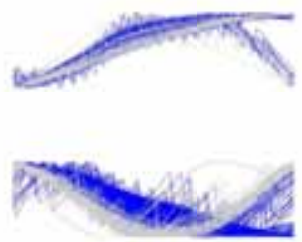

(a)
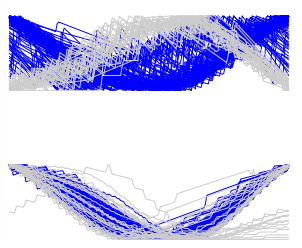

(b)

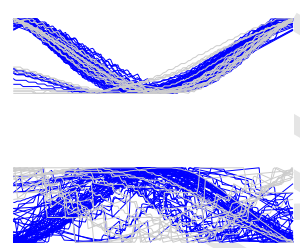

(c)

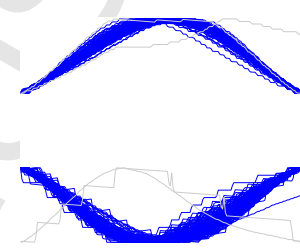

(d)

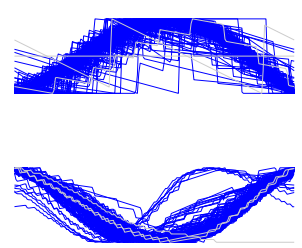

(e)

Figure 12: Measured signatures of the (a) cone, (b) torus, (c) sphere, (d) hyperboloid, (e) cylinder after been enhanced. The signatures in blue are the ones that fulfill the critiria stablished in Section 4.2 to be consired as a match of the theoretical signature. The ones in gray are the ones that do not.

measured signatures of the cylinder are therefore closer to the theoretical signature of the hyperboloid than to its corresponding one. When data obtained with real objects on a Shadow hand, we found the same behavior (Fig. 13b).

It is also observable that for most of the signatures, the mean distances between the signatures and the corresponding patterns fall below a threshold of 50, except for the sphere, which mean goes up to around 80. The threshold was fixed here to 55. In further experiments, the measured signatures of the cylinder were compared to the theoretical signature of the hyperboloid. To differentiate the signatures of both shapes, the integral obtained from the signature enhancement procedure was used. Because the cylinder has a straight shape, the integral would be lower for this shape than for the hyperboloid (Fig. 14). To differentiate a signature from another, a threshold was defined as the average of both means. This is confirmed in the experiments with the real hand. A slight difference in the threshold is found. This difference can be attributed to the amount of data gathered with real objects and the differences between the simulated model of the shadow hand and real hand.

\subsection{Ranges Definition}

Table 2 shows for each shape the range of the radius obtained when using the threshold defined in the previous section. It also shows the interval of positions in the hand considered for each shape. As can be observed, the measured signatures of the torus correspond to upper locations. This is due to the fact that given a horizontal pose of the torus in the hand, the contact zone is not located on the top of the shape as shown Fig. 8 but rather

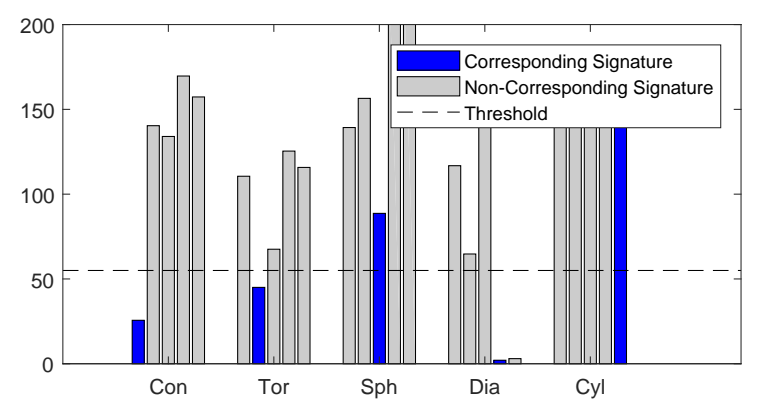

(a)

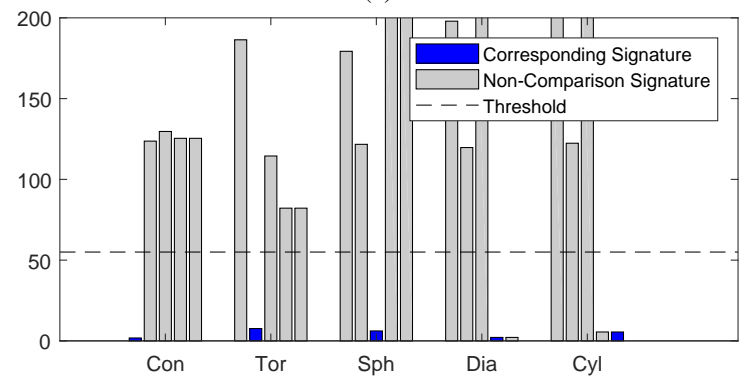

(b)

Figure 13: DTW results when the measured signatures are compared to the theoretical ones. For each shape, the signatures are compared with all patterns of the five shapes. The blue bars refer to the results obtained when the signatures are compared to their pattern corresponding and the gray bars, when they are compared to the patterns of other shapes. The dash line is the defined threshold. (a) Simulation. (b) with real hand.

in the inner part as the one shown in Fig. 15. Thus, because the shape of the torus in this location is similar to the hyperboloid, 


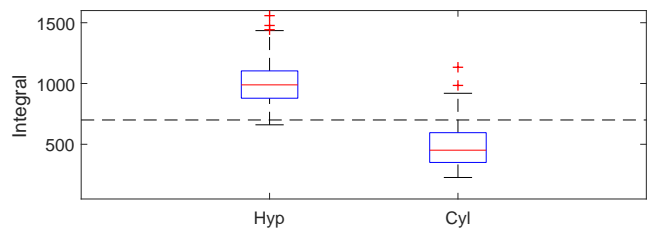

(a)

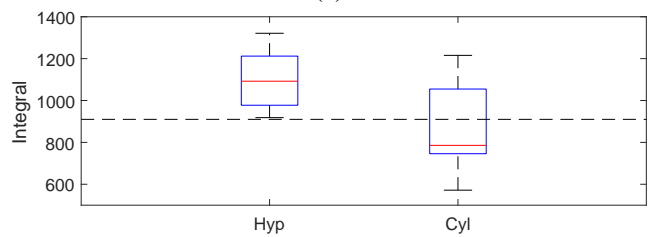

(b)

Figure 14: Integrates of the hyperboloid and cylinder resulting from the signature enhancement procedure. The dashed line is the threshold defined to distinguish the signatures of these two shapes. This threshold was computed using the mean of the mean values of the integrates of each shape. (a) Simulation. (b) with real hand.

so is the measured signature.

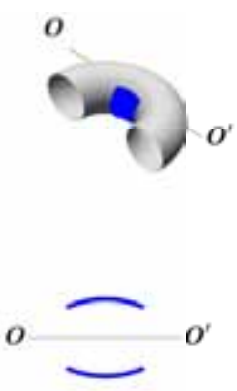

Figure 15: Illustration of the location of the manifold formed by the fingers adapting to a torus located near the palm and the its corresponding generated signature. The shape is cross-sectioned to facilitate the view.

In the experiences with real objects, their sizes and locations were chosen to fall within these ranges, which explains why results depicted in Fig. 13a show that all shapes felt below the threshold established in section 4.2.

\subsection{Rotated Objects}

In realistic situations, objects might also be rotated with respect to the hand. Experiments were done with the torus, hyperboloid and cylinder. The inclination of the objects was increased by 2.5 degrees from 5 to 25 degrees. The size and the position were changed like in the previous experiments within their ranges. Fig. 16 shows the percentage of measured signatures that correspond to the theoretical signatures as a function of the inclination angle. Furthermore, in Fig. 17, the measured angle of the signature is plotted against the actual inclination angle of the object for both (a) simulation and (b) real objects.

As shown in these results, which were obtained in simulation, the presented method succeeds in correcting the effects of the object rotation on their signatures up to an angle of $25^{\circ}$ for all shapes. Concerning real objects, results are shown in Fig. 17b. As it can be observed, a linear regression can be used to

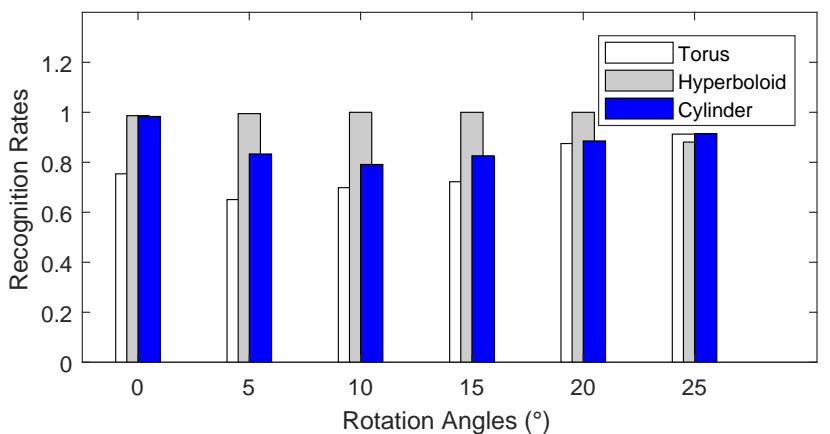

Figure 16: Identification rate of rotated objects with respect to the angle of rotation.

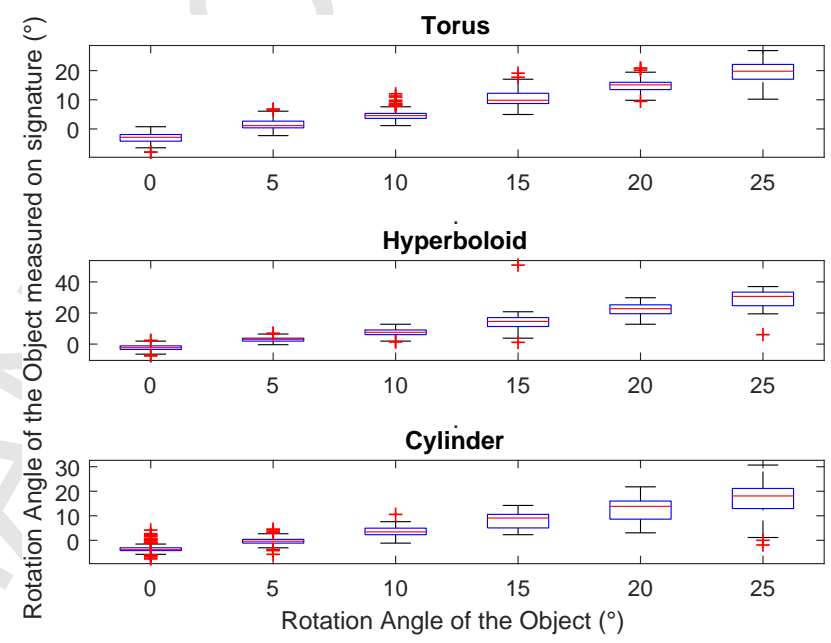

(a)

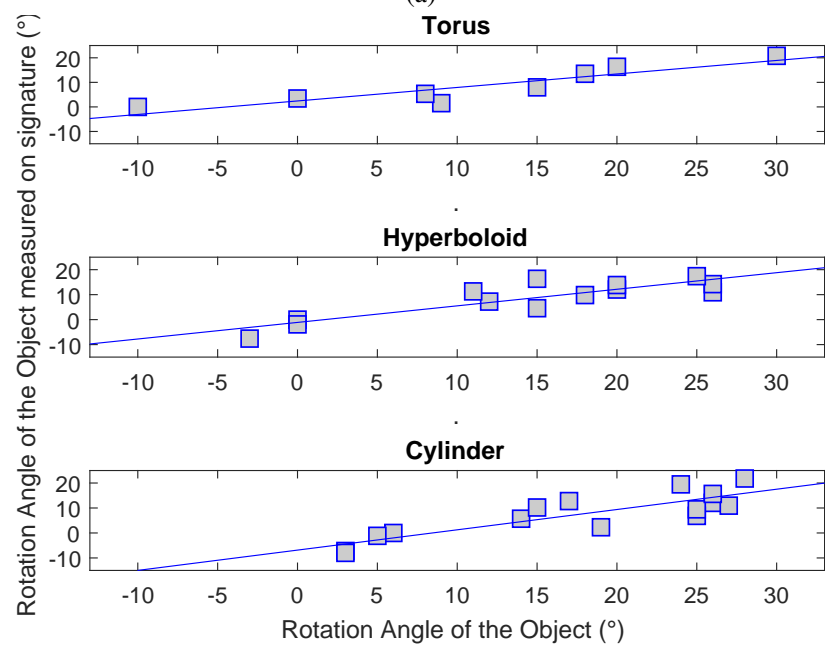

(b)

Figure 17: Measured angle of the signatures versus the actual angle of rotation of the object. (a) Simulation. (b) with real hand. The blue lines in this images were computed using linear regression.

model the behavior of the angle measurements based on the signatures for each shape. Therefore, this method offers a means to estimate the inclination of the object. This is an interesting feature because it adds another advantage to the signature, such as the capability of estimating the pose of the object within the hand. 
Table 2: Ranges of size and positions in the hand attained for each one of the shapes.

Cone Torus

\subsection{Modified Shapes}

It was desired to study the performance of the presented method for shapes similar to the ones used in previous sections. For this, an ovoid and an pyramid with an hectagon base were chosen as modifications to the sphere and cone, respectively. Results are shown in Fig. 18. As it can be observed, for the pyramid, its signature corresponded to the one of a cone, confirming what was said in section 2.1.2 about proprioception not being able to detect edges and generating the same signatures for smooth of polygonal shapes. As for the ovoid, it generated signatures similar to that of a sphere or a torus. When data was analyzed in more detail, we realized that it depended on the position of the ovoid within the hand. Thus, for lower positions, a signature similar to that of a sphere would be generated, and as the location went upwards in the hand, the signature becomes similar to that of a torus.

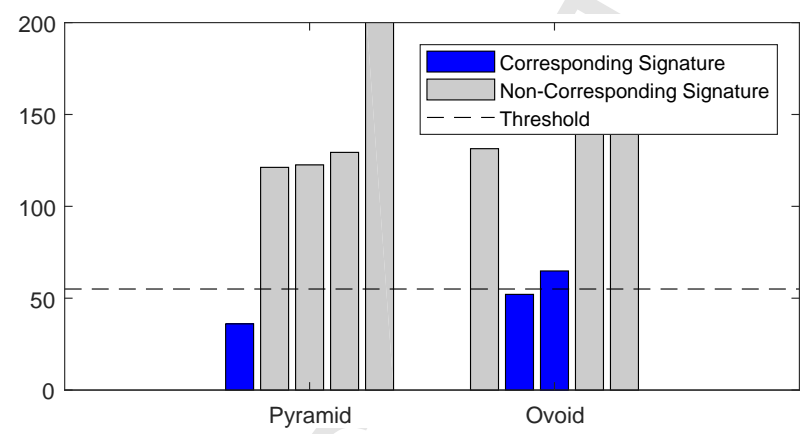

Figure 18: DTW distances computed for an ovoid and a hectagon pyramid. The theoretical signatures of a cone, torus, sphere, hyperboloid and cylinder were used.

\section{Conclusions}

In this paper, proprioceptive data was used to identify the shape of a grasped object in a robotic hand. For this task, a representation of the joint angles of the fingers was created in which the finger configurations were sketched. This representation allowed to find patterns related to the grasped shapes, thus to generate the so-called proprioceptive shape signatures. It was shown that these signatures have the potential of being profitable for both object recognition and manipulation tasks because they contain information on the global shape and pose of the object within the hand.

Experiments were carried on simulation and a comparison on results obtained on both cases were compared and showed to behave similarly. An analysis on the signatures using DTW showed that measured signatures have a significant correspondence to the theoretical one of the same shape, and a very low correspondence to those of different shapes. When shapes look similar to each other, such as cylinder and hyperboloid, and cannot be distinguished by its normalized signatures only, other parameters can be used for this. The cylinder is a at shape and the integral of its signature is lower than the integral of the signature of the hyperboloid. Thus, using both the signature and its integral, it can be concluded that they correspond exclusively to a specific shape which is beneficial for shape identification tasks.

Signatures showed little variance within large ranges of size and pose (position and orientation) for each shape since the effects of both parameters on the signatures were discarded by the procedure explained in section 2.4. This brings several key features to the presented method. First, instead of focusing on specific object recognition, signatures allow shape identification for any object within the ranges defined in Table 2. Second, the angle of inclination of the object in the hand can be estimated through the integral of the signature. Third, the signatures are generated and enhanced in about $20 \pm 2$ seconds. So, all together those object characteristics can be used in real-time for manipulation tasks where physical knowledge on the object is more important than its identification.

The shape of objects with high elasticity and exibility could not be identified with this method. However, results with real objects confirm that objects can slightly deform (like the plastic 
bottles used in experiments) and the method would still work.

In future work, the signatures will be combined with tactile information to identify fine details of the object shape, also important for manipulation tasks. An exploration algorithm based on those proprioceptive signatures will also be developed by exploiting the information about the pose of the object within the hand to control the direction of the movements of the hand.

\section{Appendix}

\subsection{Equivalence between the joint angles and central angles of grasped circle}

In the Fig. 19, points $\mathrm{O}, \mathrm{A}, \mathrm{B}$ and $\mathrm{C}$ define a quadrilateral. The sum of its internal angles is equal to $360^{\circ}$.

$$
\angle O+\angle A+\angle B+\angle C=360^{\circ}
$$

Let $l_{1}$ and $l_{2}$ be tangents to the circle $O$ at the points $A$ and $C$. Hence, the cords $\overline{O A}$ and $\overline{O C}$ are orthogonal to $l_{1}$ and $l_{2}$, respectively and

$$
\angle O+\angle B=180^{\circ}
$$

Because $\angle B$ and $\angle B$ are supplementary angles,

$$
\angle B+\angle B=180^{\circ}
$$

Substituting (2) in (3) demonstrates that

$$
\angle O=\angle B
$$

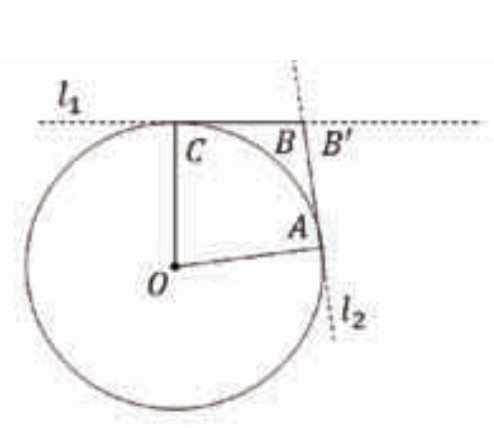

Figure 19: Tangents $l_{1}$ and $l_{2}$ and the cords $\overline{O A}$ and $\overline{O C}$ forming a quadrilateral.

\section{References}

[1] R. L. Klatzky, S. J. Lederman, V. A. Metzger, Identifying objects by touch: An expert system, Perception \& psychophysics 37 (4) (1985) 299302.

[2] B. Wang, L. Jiang, J. Li, H. Cai, Grasping unknown objects based on 3d model reconstruction, in: Advanced Intelligent Mechatronics. Proceedings, 2005 IEEE/ASME International Conference on, IEEE, 2005, pp. 461-466.

[3] H.-Y. Jang, H. Moradi, S. Lee, J. Han, A visibility-based accessibility analysis of the grasp points for real-time manipulation, in: Intelligent Robots and Systems, 2005.(IROS 2005). 2005 IEEE/RSJ International Conference on, IEEE, 2005, pp. 3111-3116.

[4] V. Lippiello, F. Ruggiero, B. Siciliano, L. Villani, Visual grasp planning for unknown objects using a multifingered robotic hand, IEEE/ASME Transactions on Mechatronics 18 (3) (2013) 1050-1059.
[5] H. Kawasaki, T. Komatsu, K. Uchiyama, Dexterous anthropomorphic robot hand with distributed tactile sensor: Gifu hand ii, Mechatronics, IEEE/ASME Transactions on 7 (3) (2002) 296-303.

[6] S. Begej, Planar and finger-shaped optical tactile sensors for robotic applications, Robotics and Automation, IEEE Journal of 4 (5) (1988) 472-484.

[7] D. Johnston, P. Zhang, J. Hollerbach, S. Jacobsen, A full tactile sensing suite for dextrous robot hands and use in contact force control, Robotics and Automation, 1996. Proceedings., 1996 IEEE International Conference on 4 (1996) 3222-3227.

[8] H. Liu, K. C. Nguyen, V. Perdereau, J. Bimbo, J. Back, M. Godden, L. D. Seneviratne, K. Althoefer, Finger contact sensing and the application in dexterous hand manipulation, Autonomous Robots 39 (1) (2015) 25-41.

[9] Z. Pezzementi, E. Plaku, C. Reyda, G. D. Hager, Tactile-object recognition from appearance information, Robotics, IEEE Transactions on 27 (3) (2011) 473-487.

[10] A. Schneider, J. Sturm, C. Stachniss, M. Reisert, H. Burkhardt, W. Burgard, Object identification with tactile sensors using bag-of-features, in: Intelligent Robots and Systems, 2009. IROS 2009. IEEE/RSJ International Conference on, IEEE, 2009, pp. 243-248.

[11] A. M. Okamura, M. R. Cutkosky, Feature detection for haptic exploration with robotic fingers, The International Journal of Robotics Research 20 (12) (2001) 925-938.

[12] H. Liu, X. Song, T. Nanayakkara, L. D. Seneviratne, K. Althoefer, A computationally fast algorithm for local contact shape and pose classification using a tactile array sensor, in: Robotics and Automation (ICRA), 2012 IEEE International Conference on, IEEE, 2012, pp. 1410-1415.

[13] S. J. Lederman, R. L. Klatzky, Hand movements: A window into haptic object recognition, Cognitive psychology 19 (3) (1987) 342-368.

[14] C. Strub, F. Worgotter, H. Ritter, Y. Sandamirskaya, Correcting pose estimates during tactile exploration of object shape: a neuro-robotic study, in: Development and Learning and Epigenetic Robotics (ICDL-Epirob), 2014 Joint IEEE International Conferences on, IEEE, 2014, pp. 26-33.

[15] N. Gorges, S. E. Navarro, D. Göger, H. Wörn, Haptic object recognition using passive joints and haptic key features, in: Robotics and Automation (ICRA), 2010 IEEE International Conference on, IEEE, 2010, pp. 2349_ 2355 .

[16] M. Jin, H. Gu, S. Fan, Y. Zhang, H. Liu, Object shape recognition approach for sparse point clouds from tactile exploration, in: Robotics and Biomimetics (ROBIO), 2013 IEEE International Conference on, IEEE, 2013, pp. 558-562.

[17] B. Browatzki, V. Tikhanoff, G. Metta, H. H. Bülthoff, C. Wallraven, Active object recognition on a humanoid robot, in: Robotics and Automation (ICRA), 2012 IEEE International Conference on, IEEE, 2012, pp. 20212028.

[18] A. Bierbaum, K. Welke, D. Burger, T. Asfour, R. Dillmann, Haptic exploration for $3 \mathrm{~d}$ shape reconstruction using five-finger hands, in: Humanoid Robots, 2007 7th IEEE-RAS International Conference on, IEEE, 2007, pp. $616-621$.

[19] J. Sinapov, T. Bergquist, C. Schenck, U. Ohiri, S. Griffith, A. Stoytchev, Interactive object recognition using proprioceptive and auditory feedback, The International Journal of Robotics Research 30 (10) (2011) 12501262.

[20] S. R. Company, Shadow dexterous hand TM, http://www.shadowrobot.com/products/dexterous-hand/ (2017).

[21] P. S.R.L., Robotic forearms, http://www.prensilia.com (2016).

[22] K. Kaneko, K. Harada, F. Kanehiro, Development of multi-fingered hand for life-size humanoid robots, in: Proceedings 2007 IEEE International Conference on Robotics and Automation, 2007, pp. 913-920.

[23] D. R. Faria, J. Lobo, J. Dias, Identifying objects from hand configurations during in-hand exploration, in: Multisensor Fusion and Integration for Intelligent Systems (MFI), 2012 IEEE Conference on, IEEE, 2012, pp. 132-137.

[24] M. Johnsson, C. Balkenius, Experiments with proprioception in a selforganizing system for haptic perception, Proceedings of TAROS 2007 (2007) 239-245.

[25] S. Ratnasingam, T. M. McGinnity, Object recognition based on tactile form perception, in: Robotic Intelligence In Informationally Structured Space (RiiSS), 2011 IEEE Workshop on, IEEE, 2011, pp. 26-31.

[26] T. Bergquist, C. Schenck, U. Ohiri, J. Sinapov, S. Griffith, A. Stoytchev, Interactive object recognition using proprioceptive feedback, in: Proceedings of the 2009 IROS Workshop: Semantic Perception for Robot Manip- 
ulation, St. Louis, MO, 2009.

[27] S. Gallagher, J. Cole, Body image and body schema in a deafferented subject., Journal of mind and behavior.

[28] S. J. Lederman, R. L. Klatzky, Haptic perception: A tutorial, Attention, Perception, \& Psychophysics 71 (7) (2009) 1439-1459.

[29] J. Voisin, Y. Lamarre, C. E. Chapman, Haptic discrimination of object shape in humans: contribution of cutaneous and proprioceptive inputs, Experimental Brain Research 145 (2) (2002) 251-260.

[30] J. R. Napier, The prehensile movements of the human hand, Journal of bone and joint surgery 38 (4) (1956) 902-913.

[31] F. N. Newell, M. O. Ernst, B. S. Tjan, H. H. Bülthoff, Viewpoint dependence in visual and haptic object recognition, Psychological science 12 (1) (2001) 37-42.

[32] R. Bellman, R. Kalaba, On adaptive control processes, IRE Transactions on Automatic Control 4 (2) (1959) 1-9. 


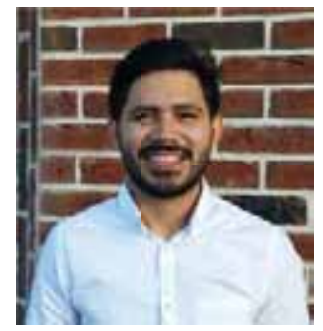

Alex VASQUEZ is a PhD candidate at UPMC since 2014. He received the B.E. degree in electrical engineering from the University of Zulia, Venezuela, in 2010, and the MS. In Advance Robotics with a focus on rehabilitation robotics from the University Pierre and Marie Curie (UPMC), Paris, France in 2014. With his main career interests focused on robotics and research, he participated in national and international IEEE robotics competition, he worked as professor's assistant during his undergraduate studies and he actively participated in the research on electromagnetic compatibility in his university. He is now doing his research regarding the tactile object recognition in a robotic hand at the Institute of Intelligent Systems and Robotics (ISIR). 


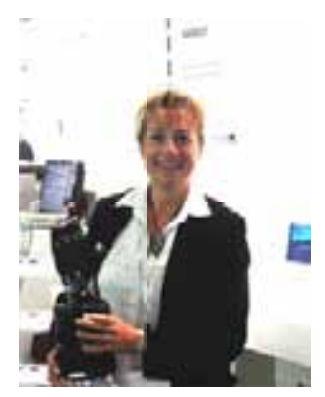

Prof. Véronique PERDEREAU is full professor at UPMC since 2003 and an IEEE senior member. She obtained her Electrical and Information engineering MS in 1987 and Robotics and Automation $\mathrm{PhD}$ degree in 1991. She is author of more than 100 scientific published papers. She has organized several international conference sessions and workshops, reviewed for many international publications and has been a member of several scientific program committees. She has been invited to several international conferences. She is principal investigator in several collaborative EU projects. She received in 2013 from the French Ministry of Research and Education the price "Etoiles de l'Europe" for the coordination of the HANDLE EU project and was appointed Knight of Legion of Honor in 2014. 
- A descriptor based in proprioception is developed for shape identification

- Descriptor allows estimating rotation and shape of the object

- Temporal analysis of proprioceptive allows determining fingers in contact

- Objects are identified regardless its size and position within the hand

- The proprioceptive descriptors could be used in real-time applications 